In Crescendo. Institucional. 2015; 6(1): 235-246

\title{
HiPÓTESIS DE INCIDENCIA TRIBUTARIA
}

\section{HYPOTHESIS OF TAX INCIDENCE}

\author{
Dalil O. Zapata Cruz
}

\section{RESUMEN}

En $_{\mathrm{p}}^{\mathrm{n}}$ todo impuesto debe señalarse la hipótesis de incidencia tributaria, que es el supuesto de hecho definido en forma abstracta por la ley, que llevado a cabo por un sujeto designado por ella, en el lugar determinado por ella, genera la obligación de pagar el tributo. Es necesario detallar su norma matriz, la obligación tributaria propiamente entrelazada con el hecho imponible e hipótesis de incidencia tributaria en sí con sus respectivos aspectos.

Palabras claves: hecho imponible, hipótesis de incidencia, tributo, obligación tributaria, contribuyente.

\begin{abstract}
The hypothesis of incidence tax, pointing out what the course actually defined in abstract form by law, carried out by an individual designated by it, in the place determined by the same, it generates the obligation to pay the tribute should be noted in any tax. It is necessary to specify its parent rule, the tax obligation itself intertwined with taxable and tax incidence itself with its respective aspects hypothesis.
\end{abstract}

Keywords: taxable, hypothesis of incidence, tribute, tax, taxpayer obligation.

* Bachiller en Educación en Ciencias Religiosas y estudiante del octavo ciclo de la carrera profesional de Derecho y Ciencia Política en la Universidad Católica Los Ángeles de Chimbote. Correo electrónico: odinvirjustus@gmail.com 


\section{HiPÓTESIS DE INCIDENCIA TRIBUTARIA}

En la época del antiguo Egipto, los faraones otorgaron muchos tributos a los escribas. En un periodo, los escribas impusieron un tributo en el aceite de cocina. Para asegurarse de que los ciudadanos no burlaran el tributo, los escribas auditaban las cantidades apropiadas de aceite de cocina que aquellos consumían y fiscalizaban que no usaran residuos generados por otros procesos de cocina como sustitutos para el aceite gravado.

La organización económica era tributaria. ¿Qué significa esto? Significa que los súbditos estaban obligados a entregar al faraón una cierta cantidad de mercancías o trabajo personal (el tributo sería una especie de impuesto). Así surge el tributo desde el hecho mismo de la imposición que determinará su rol en la obligación tributaria desde su fundamento en la hipótesis de incidencia tributaria.

Así, los tributos eran utilizados para comerciar con otras regiones, para pagar a los funcionarios del Estado, para repartir en épocas de hambruna y para financiar la construcción de obras públicas.

Con el transcurrir del tiempo, los elementos propios de la hipótesis de incidencia tributaria se modernizarán, pero sin perder el fin de la misma obligación tributaria.

Por ello es necesario dilucidar sobre la hipótesis de incidencia tributaria.

\section{La norma matriz de incidencia tributaria}

\subsection{La norma tributaria}

Para el doctor Ataliba, G. (1987, p. 55), "el derecho tributario objetivo es el conjunto de normas jurídicas que regula la tributación, entendiéndose por tributación la acción estatal de tributar".

Por tanto, al tratar sobre la tributación, es necesario señalar la norma tributaria que constituye desde su primera aparición el conjunto de reglas que debe cumplir un agente capaz en proporción al sistema cultural que se desarrolla, específicamente en el campo democrático.

Por ello no es admisible desde el campo cognitivo y racional el hecho de hacer notar la posibilidad de que exista un agente capaz que no sea consciente de su cumplimiento. Eso desvirtúa el sentido racional a la vez que perjudica a la sociedad, que reprueba y actúa desde el Estado contra la informalidad y todo tipo de evasión, elusión, contrabando y faena o labor no registrada, los cuales demuestran claramente que no se ha instalado adecuada o realmente una cultura fiscal que vele por salvaguardar aquellos intereses propios de cada agente capaz, razón por la se usa en forma debida la afectación principal.

\subsection{Tipología}


Es un modelo de la estructura propiamente dicha de la relación tributaria que puede ser norma primaria, así como a su vez puede ser norma secundaria. En el primer caso se menciona claramente su naturaleza sensu stricto y en el segundo caso se determina su función fiscalizadora.

\subsection{Concepto de incidencia}

Según el doctor De Barros, P. et al. (2003, p. 559), es "la técnica del derecho, es su modo de referirse a los objetos y situaciones objetivas a través del pensamiento o hipótesis fáctica de la norma".

Este texto señala que sería la incidencia propiamente dicha todo aquel fenómeno o hecho propio perteneciente a la norma jurídica. La norma matriz de la incidencia tributaria consiste en obtener de la norma tributaria tanto la hipótesis de incidencia como la consecuencia normativa de lo que se pretende gravar.

Por tanto, la incidencia tributaria será un resultado del proceso de transferencia o traslación de impuestos. Cuando hay un proceso de transferencia de impuestos, este puede ser hacia atrás o hacia adelante.

Esto implica, según el doctor Ataliba (1987, p. 61) que “[...] supone la regla jurídica y el hecho o hechos sobre los cuales ella incida, volviéndolos hechos jurídicos".

Es la relación de coexistencia entre el hecho real con el supuesto hecho descrito en la disposición legal.

La incidencia tributaria definida en términos generales comprende el estudio de los efectos de la política tributaria sobre la distribución del bienestar económico. La colocación de impuestos afecta al precio de los bienes o la retribución a los factores de producción. Para evaluar la incidencia tributaria se tienen que conocer los efectos de esta sobre los distintos mercados y agentes económicos.

La incidencia tributaria comprende varias dimensiones, tales como los efectos de los impuestos en la distribución del ingreso de los factores, sobre el grado de desigualdad del ingreso, sobre el bienestar intergeneracional y sobre los consumidores de diferentes productos.

El doctor De Barros, P. et al. (1999, p. 561) estipula:

"Se aprecia que la llamada incidencia jurídica se reduce, por el prisma lógico, a dos operaciones formales: la primera, de subsunción o de inclusión de clases... se incluye en la clase de los hechos previstos y en el supuesto de la norma general y abstracta: ... la segunda, de implicación, por cuanto la fórmula normativa prescribe que el antecedente implica la tesis...".

Es necesario entender entonces que entre los dos aspectos determinados, uno de ellos es 'juridizar' el hecho y lograr que este mismo hecho genere efectos de índole jurídica. Por lo tanto, cabe aseverar que su eficiencia está en producir necesariamente efectos jurídicos. 
La incidencia tributaria de un impuesto tiene implicancias directas en la distribución del ingreso, y, por lo tanto, en la distribución del bienestar económico. Además, el gobierno usa la recaudación tributaria para entregar beneficios a los ciudadanos, lo cual es un segundo impacto distributivo asociado a los impuestos, generalmente más importante que el primero.

\subsection{La fórmula de la regla matriz}

El doctor De Barros, P. (2003, p. 560) estipula que "la norma matriz es la parte de la norma tributaria que contiene: la hipótesis de incidencia o descripción del hecho que se pretende gravar y la consecuencia normativa que se atribuye a la realización del hecho descrito en la hipótesis".

Por ende, la fórmula de la hipótesis de incidencia tributaria es $\mathrm{H}=\mathrm{A}-(\mathrm{B}+\mathrm{C})$

$\mathrm{H}=$ Hipótesis de incidencia

A $=$ Hechos tributables

$\mathrm{B}=$ Hechos inmunes e inafectos

$\mathrm{C}=$ Hechos exentos

\section{La obligación tributaria}

\subsection{El tributo}

En relación con el objeto de la obligación tributaria, el doctor Ataliba, G. (1987, p. 55) señala que es la "[...] transferencia de [...] dinero [...] contempla el concepto de tributo la noción de sujeto pasivo, como siendo alguien sometido a la ley, y la de sujeto activo, como persona pública, por lo general".

Si bien es cierto que en la legislación peruana no existe el concepto de 'tributo', el derecho comparado nos señala que son las prestaciones pecuniarias o los ingresos públicos de carácter obligatorio impuesto por la administración pública como consecuencia directa de la realización del hecho imponible al que la ley vincule en el deber de contribuir.

El fin del tributo, de la misma manera que en época de los egipcios, es obtener ingresos de carácter imprescindible para sostener el gasto público.

\subsection{La obligación tributaria}

La doctora Robles, C. (2013, p. 2) en "Introducción a la obligación tributaria" sostiene que "es el vínculo entre el acreedor y el deudor tributario, establecido por la ley, que tiene por objeto el cumplimiento de la prestación tributaria, siendo exigible coactivamente".

Esa obligación nace en el hecho mismo de estar prevista en la ley, única fuente generadora de esa obligación.

La obligación es de por sí un vínculo de naturaleza jurídica que contiene la prestación pecuniaria preferentemente. Por tanto, el sujeto o contribuyente debe pagar la deuda 
tributaria, caso contrario la administración pública tiene las facultades y los derechos para exigirle su cumplimiento.

La obligación tributaria nace cuando se realiza el hecho previsto en la ley, como generador de esa obligación tributaria.

O como lo estipuló la Facultad de Derecho de la Universidad de San Martín de Porres (1996, p. 16): "no nace de un acuerdo de voluntades, sino del producto de la relación entre la hipótesis de incidencia y la realidad económico-fiscal, y como consecuencia de haberse verificado un hecho imponible".

\subsubsection{Determinación de la obligación tributaria}

Es el acto emanado de la misma administración, de los particulares o de ambos coordinadamente, con el fin de fijar el hecho imponible y, por ende, el alcance directo de la obligación. Por la determinación de la obligación tributaria, el Estado provee la ejecución efectiva de su pretensión.

Como sabemos, la ley establece en forma general las circunstancias o los presupuestos de hecho, de cuya producción deriva la sujeción al tributo. Este mandato indeterminado (supuesto hipotético, abstracto) tiene su secuencia en una operación posterior, mediante la cual la norma de la ley se particulariza, se individualiza y se adapta a la situación de cada persona que pueda hallarse incluida en la hipótesis de incidencia tributaria.

En este orden de ideas, la situación hipotética contemplada por la ley se concreta y exterioriza en cada caso particular. En materia tributaria -insistimos- este procedimiento se denomina determinación de la obligación tributaria.

Es el procedimiento por el que el tributarista verifica la realización del hecho que genera la misma obligación tributaria, señala la base imponible y la cuantía del tributo en sí.

\subsubsection{Clases de obligación}

\section{Sustancial}

Prestación de índole patrimonial declarada en la obligación de dar por parte del contribuyente, así como del de recibir por el fisco.

\section{Formal}

Aparece en la interrelación entre sujeto activo y pasivo de la obligación tributaria con el objetivo de facilitar su cumplimiento tributario.

\subsubsection{La declaración tributaria}

Es el hecho de transmitir información a la administración del área tributaria, propio del sujeto pasivo. La administración se comunica con el administrado mediante actos administrativos llamados declaraciones, que no es más que la misma transmisión de la información. 


\subsection{El hecho generador}

La misma doctora Robles, C. (2013, p. 1) señala que es el "presupuesto establecido por la ley para tipificar el tributo, cuya realización origina el nacimiento de la misma obligación". Se le conoce con otros nombres, entre los cuales tenemos hecho gravable, hecho tributario, hecho gravado, presupuesto de hecho, falto impositivo, fato gerador.

El hecho generador da nacimiento a la obligación tributaria, pues la ley por sí sola no puede cumplir con este fin; esto obedece a que la norma no puede indicar dentro de su ordenamiento a los deudores individuales del tributo, por tal razón se sostiene del hecho imponible, que al realizarse determina el sujeto pasivo y la prestación a que está obligado.

Se estima llamarse así por su amplia significación, se ajusta mejor a la situación que trata de configurarse, además de ser corriente de opinión en varios países, especialmente en América Latina.

\section{La hipótesis de incidencia tributaria}

\subsection{Concepto legal}

Según el doctor Ataliba, G. (1987, p. 66), "es [...] la descripción legal de un hecho; es la formulación hipotética, previa y genérica, contenida en la ley, de un hecho".

Como sabemos, toda norma contiene una hipótesis y un mandato, que solo es obligatorio asociado a la hipótesis. Por su parte, la hipótesis de la norma describe los actos abstractos que, siempre y cuando acontezcan, convierten el mandato en obligatorio; asimismo describe las cualidades de las personas que deberán tener el comportamiento prescrito en el mandato.

\subsection{Carácter universal}

El doctor Ataliba, G. (1987, p. 68) señala que "su concepto es universal, en el sentido de que no deriva de la observación de cualquier sistema, ni se compromete con ningún instituto jurídico real".

Este texto manifiesta que el criterio de la hipótesis de la incidencia tributaria no es propiedad exclusiva de un sistema jurídico en sí, sino más bien es universal, para todos los tiempos y para todos los espacios en cualquier sistema jurídico implantado. Por su característica de imponible, la obligación tributaria y por ser todo un conexo de tratados y estudios internacionales en su debida ejecución mundial.

\subsection{Unidad lógica}

El mismo doctor Ataliba, G. (1987, p. 73) señaló que "la principal consecuencia de la unidad de la hipótesis de incidencia está en que el hecho imponible es también unitario e inescindible (postulado metodológico y axiomático) que determina el nacimiento de una obligación tributaria". 
Es una e inescindible esta hipótesis porque va a unir los elementos propios y diversos conjugando los términos. Por este medio se califica la habilitación del nacimiento de los tributos y su carácter obligatorio.

La consecuencia inmediata y necesaria del carácter unitario de la hipótesis de incidencia está, en cada caso, en identificar cada hipótesis de incidencia solo consigo misma, y en el plano epistemológico-jurídico. Como consecuencia necesaria, cada hipótesis de incidencia solo es igual a sí misma y, por tanto, inconfundible con todas las demás.

\subsection{Aspectos}

El concepto de 'hipótesis' lleva implícitos cuatro aspectos: subjetivo, objetivo, temporal y espacial.

Sus elementos son, según el doctor De Barros, P. et al. (2003.p. 559): elemento material, personal, espacial y temporal.

Así, el aspecto subjetivo de la norma se encarga de señalar quién es el sujeto obligado a cumplir la prestación tributaria, y generalmente responde a la pregunta ¿quién es el sujeto obligado al pago?

El aspecto objetivo o material de la norma precisa qué se está afectando o gravando con la disposición, y generalmente responde a la pregunta ¿qué es lo que se grava?

El criterio material, según lo prescribe la Academia Internacional de Derecho Tributario (2001, p. 268), es "la acción que precisa realizar un sujeto para que exista presupuesto fático; y esta es el presupuesto para la producción de las normas individuales y concretas que documentan la incidencia tributaria".

El aspecto temporal de la norma nos indica el momento en el cual nace la obligación tributaria, y generalmente responde a la pregunta ¿cuándo nace la obligación?

Finalmente, el aspecto espacial precisa el lugar donde se desarrolla la operación, y generalmente responde a la pregunta ¿dónde se configura el hecho imponible?

La hipótesis de la norma describe los actos abstractos siempre y cuando acontezcan, y convierten al mandato en obligatorio; asimismo, describe las cualidades de las personas que deberán tener el comportamiento prescrito en el mandato.

Por lo tanto, según el doctor Vargas, E. (2004, p. 3), "la creación normativa de un tributo implica que su hecho imponible debe contener en forma indispensable diversos elementos: a) la descripción objetiva de un hecho o situación, b) los datos necesarios para individualizar la persona que debe realizar el hecho..., c) el momento..., d) el lugar... de la realización del hecho imponible".

La hipótesis de incidencia se sirve de coordenadas espacio-fáctico-temporales y subjetivas para describir en abstracto el hecho que el legislador desea afectar, las que, a su vez, sirven para identificar el hecho concreto que devendrá en imponible bajo el efecto de la incidencia de la norma tributaria. 
Es entonces la descripción legislativa (necesariamente hipotética) de un hecho a cuya ocurrencia en concreto la ley atribuye la fuerza jurídica de determinar el nacimiento de la obligación tributaria.

La consecuencia tributaria vendría a ser la relación obligatoria de pagar, donde el sujeto activo puede exigir a otro (sujeto pasivo) el pago de un determinado monto o prestación, con sus variables sujetos y el monto a pagar.

\section{El hecho imponible}

Es una situación descrita en la disposición legal, que al concretarse va a originar la obligación tributaria, y como consecuencia el efecto que la obligación persigue: el pago de una prestación pecuniaria.

\subsection{Sujeto}

Se refiere a la persona, al individuo que realiza la hipótesis descrita en la disposición legal.

\subsection{Sujeto activo}

El doctor Robles, C. (2006, p. I-1) señaló que "el sujeto acreedor de la obligación tributaria es la persona a quien la ley le atribuye la exigibilidad del tributo. Solo la ley puede designar el sujeto activo, como hemos señalado anteriormente. Esta designación del sujeto activo compone el aspecto personal de la hipótesis de la incidencia tributaria".

Este sujeto activo puede ser personas naturales, personas jurídicas, sociedades conyugales, la sucesión, la persona sin personería jurídica, los fideicomisos, los fondos mutuos de inversión en valores y los fondos de inversión. En fin, es aquel que tiene la capacidad tributaria.

\subsection{Atribución al sujeto pasivo}

El sujeto pasivo es aquel que está obligado a cumplir siempre con la misma prestación en pro del activo.

Las normas de carácter tributario tienen el supuesto denominado hipótesis de incidencia tributaria; y la consecuencia es la obligación tributaria, dentro de la cual encontramos al sujeto pasivo.

El doctor Jarach, D. (2002, p. 177) manifiesta que "solamente el sujeto pasivo principal, el deudor por título propio o contribuyente puede ser determinado sin necesidad de alguna norma expresa por parte de la ley, porque se deduce de la naturaleza del hecho imponible, esté o no indicado en una norma explícita del derecho tributario material".

El sujeto pasivo es la persona legalmente obligada a pagar el impuesto a la administración tributaria. Todos los impuestos, y también las retenciones o pagos a cuenta que 
puedan establecerse, tienen un sujeto pasivo; sin embargo no siempre será quien haya de soportar el impuesto.

La condición de sujeto pasivo recae en general en todas las personas naturales y jurídicas o entes a los cuales el derecho tributario les atribuya la calidad de sujeto de derechos y obligaciones.

Cabe distinguir entre el contribuyente, al que la ley impone la carga tributaria por ser el titular de la capacidad de pago que el impuesto pretende gravar, y el responsable legal o sustituto del contribuyente, que estará obligado al cumplimiento formal y material de la obligación tributaria, aunque puede darse la coincidencia de ambos.

Cuando se produce esta disociación legal entre la persona formalmente obligada a pagar el impuesto (sujeto pasivo) y aquella otra que ha soportado legalmente, a esta última suele llamarse el contribuyente, es decir, la persona que paga los impuestos al Estado.

\subsection{El contribuyente}

El doctor Jarach, D. (2002, p. 168) estipuló que el contribuyente "está obligado al pago del tributo por un título propio, y, si se permite decir, por naturaleza, porque respecto a él se verifica la causa jurídica del tributo".

En la ley es necesario que se presenten los criterios propios para determinar los casos de tributación, los momentos, las identificaciones del pasivo y la cuantía debida. Por tanto, los impuestos personales son los hechos imponibles que tienen importancia de cualidades personales del contribuyente. Los impuestos son indiferentes a las cualidades propias del contribuyente propiamente dicho.

El sujeto pasivo es la persona legalmente obligada a pagar el impuesto a la administración tributaria. Todos los impuestos, y también las retenciones o pagos a cuenta que pueda establecerse, tienen un sujeto pasivo; sin embargo, no siempre será quien haya de soportar el impuesto.

El párrafo 1 del artículo 4 especifica que la condición de sujeto pasivo recae en general en todas las personas naturales y jurídicas o entes a los cuales el derecho tributario les atribuye la calidad de sujeto de derechos y obligaciones.

Cabe distinguir entre el contribuyente, al que la ley impone la carga tributaria por ser el titular de la capacidad de pago que el impuesto pretende gravar, y el responsable legal o sustituto del contribuyente, que estará obligado al cumplimiento formal y material de la obligación tributaria, aunque puede darse la coincidencia o no de ambos.

Cuando se produce esta disociación legal entre la persona formalmente obligada a pagar el impuesto (sujeto pasivo) y aquella otra que ha soportado legalmente, a esta última suele llamarse el contribuyente, es decir, la persona que paga los impuestos al Estado. 


\subsection{El responsable}

Según el doctor Vargas, E. (2004, p. 5), el responsable "no es el directamente obligado pero debe cumplir la obligación tributaria porque así lo manda la ley".

El texto citado quiere decir que el responsable del tributo es el también llamado sujeto pasivo de hecho, que es la persona jurídica o natural (a diferencia del contribuyente, que puede ser otro tipo de sujeto activo) que está directamente vinculada al pago de la prestación pecuniaria establecida según los cánones de la ley.

Se encuentra obligado al cumplimiento de la prestación tributaria que se ha generado por la ocurrencia de un hecho realizado por otro sujeto denominado 'contribuyente'. Cabe indicar que la responsabilidad tributaria regulada es solidaria, es decir, la administración tributaria, una vez determinada la responsabilidad tributaria, puede cobrar indistintamente al contribuyente o al responsable (o a ambos).

\subsection{Capacidad jurídica tributaria}

El doctor Jarach, D. (2002, p. 119) estipuló que la capacidad jurídica tributaria "es la apreciación por parte del legislador de que el hecho económico se ha verificado y permite al sujeto distraer una suma de dinero de sus necesidades privadas para destinarla a contribuir los gastos públicos".

Se le declara así como el principio de antonomasia que ha podido alcanzar tal categoría logrando su obtención constitucional de manera explícita y real en un sinnúmero de ordenamientos o sistemas jurídicos existentes.

Consiste claramente en la capacidad económica de la que dispone el sujeto activo o el contribuyente propiamente dicho en el campo tributario para afrontar prestaciones de carácter coactivo y brindar sostén al gasto público para la existencia del Estado y sus necesidades. Tal capacidad deviene del hecho imponible propiamente establecido, y es resolutorio en la condición de ser indispensable su atribución al contribuyente.

El Comité Científico Asesor-Asociación Argentina de Estudios Fiscales (2003, p. 14) señaló su contemplación desde tres perspectivas: “... fundamento, ... medida, ... límite....".

a) Como fundamento ético-jurídico del deber de contribuir, deber indispensable para la existencia del Estado y la efectiva garantía de la libertad del ciudadano.

b) Como base de medida, a partir de la cual habrá de fijarse la carga fiscal concreta que deberá soportar cada contribuyente.

c) Como límite infranqueable de la potestad tributaria normativa.

\subsection{Responsabilidad tributaria}

La responsabilidad tributaria puede ser asumida tanto directa (solidaria) como indirectamente (vía sucesión, sustitución o representación). 
La Facultad de Derecho de la Universidad de San Martín de Porres (1996, p. 104) estipuló que "se trata de una institución típica del derecho tributario, según la cual la ley con el propósito de asegurar la normal recaudación de los tributos dispone el traslado del cumplimiento de la obligación hacia terceras personas distintas al contribuyente".

La responsabilidad tributaria encuentra fundamentos en la garantía y el aseguramiento del crédito tributario. Siendo este fuente del financiamiento del Estado, el legislador ha entendido que en determinados casos se designen responsables que atendiendo a algún vínculo con el contribuyente y con mayor o menor cercanía con el hecho generador de la obligación tributaria, se lo llame a responsabilidad tributaria, aumentando el número de obligados. Es palmario que es fundamento la comodidad y facilidad para el Estado en el cobro de sus créditos fiscales, pues en muchos casos se achica el campo de sujetos a fiscalizar. Se restringe el número de sujetos pasivos a controlar y constituye un instrumento para combatir la evasión.

\subsubsection{Responsabilidad directa (solidaria)}

Responsabilidad directa es el hecho mismo de asumir la prestación que coacciona al sujeto en sí.

\subsubsection{Responsabilidad indirecta}

\section{- Sucesoria}

Cuando una persona se coloca en lugar de otra respecto a las obligaciones de la relación impositiva.

\section{- Sustitutoria}

Aceptación de terceros llamados o perceptores o retenedores que se convierten en sustitutorios del contribuyente si no cumplen con la disposición legal.

\section{- Representativa}

Cuando por ley se designa al representante, cuando surge de elección voluntaria. 


\section{REFERENCIAS BIBLIOGRÁFICAS}

Ataliba G. (1987). Hipótesis de incidencia tributaria. Lima: Instituto Peruano de Derecho Tributario.

De Barros P. et al. (2003). Tratado de derecho tributario. Lima: Palestra Editores.

Robles C. (2013). Introducción a la obligación tributaria. Recuperado de: http://blog. pucp.edu.pe/item/19488/introduccion_a_la_obligacion_tributaria.

Facultad de Derecho de la Universidad de San Martín de Porres.(1996). La relación jurídico-tributaria. Vox Juris. 6. ${ }^{a}$ ed. Lima: USMP.

Academia Internacional de Derecho Tributario. (2001). Derecho tributario: tópicos contemporáneos. Lima: Grijley.

Vargas E. (2004). El hecho imponible en el Código Tributario Peruano. Recuperado de: http://roma20022.tripod.com/Trabajos_Monograficos/HECHOIMPONIBLE_PERU.

Jarach D. (2002). El hecho imponible. 3. ${ }^{\mathrm{a}}$ ed. Buenos Aires: Abeledo-Perrot.

Asociación Argentina de Estudios Fiscales. (2003). Tratado de tributación. Tomo I. Buenos Aires: Astrea. 